\title{
Study Day of Microbiology Susceptibility
}

National Cancer Institute

\section{Source}

National Cancer Institute. Study Day of Microbiology Susceptibility. NCI Thesaurus. Code C87929.

The day that a microbiology susceptibility assessment is performed. 